\title{
Trivium
}

Revue franco-allemande de sciences humaines et sociales - Deutsch-französische Zeitschrift für Geistesund Sozialwissenschaften

$5 \mid 2010$

Sociologie des conventions

\section{La sociologie des conventions. La théorie des conventions, élément central des nouvelles sciences sociales françaises}

Rainer Diaz-Bone (Université de Lucerne) et Laurent Thévenot (EHESS Paris)

Traducteur : Isabelle Dujet

\section{OpenEdition}

\section{Journals}

Édition électronique

URL : http://journals.openedition.org/trivium/3626

DOI : $10.4000 /$ trivium.3626

ISSN : 1963-1820

Éditeur

Les éditions de la Maison des sciences de l'Homme

Référence électronique

Rainer Diaz-Bone (Université de Lucerne) et Laurent Thévenot (EHESS Paris), « La sociologie des conventions. La théorie des conventions, élément central des nouvelles sciences sociales françaises », Trivium [En ligne], 5 | 2010, mis en ligne le , consulté le 08 septembre 2020. URL : http:// journals.openedition.org/trivium/3626; DOI : https://doi.org/10.4000/trivium.3626

Ce document a été généré automatiquement le 8 septembre 2020

\section{(c) (i) ()}

Les contenus des la revue Trivium sont mis à disposition selon les termes de la Licence Creative Commons Attribution - Pas d'Utilisation Commerciale - Pas de Modification 4.0 International. 


\title{
La sociologie des conventions. La théorie des conventions, élément central des nouvelles sciences sociales françaises
}

\author{
Rainer Diaz-Bone (Université de Lucerne) et Laurent Thévenot (EHESS \\ Paris) \\ Traduction : Isabelle Dujet
}

1 Cet article introduit un élément central de ce qu'on appelle «les nouvelles sciences sociales françaises ${ }^{1}$ » et vise à promouvoir une plus large réception au sein des sciences sociales germanophones des dernières évolutions de ce mouvement en France. La théorie des conventions est un mouvement transdisciplinaire, dont l'innovation consiste à créer un lien entre une économie institutionnaliste et une sociologie pragmatique. En France, cette approche s'est mise en place par le biais d'une collaboration entre des économistes, des historiens et des sociologues afin d'apporter une solution aux problèmes soulevés par l'analyse des institutions et organisations économiques et de l'action (pas uniquement) économique. La mise en œuvre du concept de convention permet d'apporter des réponses nouvelles à ces questions. En France, cette approche est qualifiée aussi d' «économie des conventions » que l'on désignera par la suite par l'abréviation $\mathrm{EC}$, ce qui ne doit cependant pas amener à penser que l'EC est simplement une partie des sciences économiques au sens étroit du terme (même si elle est désormais, en France, une approche institutionnaliste établie). Bien que l'EC ait un caractère transdisciplinaire, elle exerce une grande influence en sociologie (particulièrement en sociologie économique) et c'est la raison pour laquelle le présent numéro de TRIVIUM porte le titre de «Sociologie des conventions ».

2 Cet article aborde dans sa première partie l'évolution de la théorie des conventions, puis présente en deuxième partie les principaux concepts et en troisième partie les principaux domaines de recherche. Viennent ensuite des informations sur les traductions et des réflexions sur l'état de la réception en quatrième partie. Et pour finir 
la cinquième partie présente des remarques introductrices aux quatre articles de ce numéro de TRIVIUM. Bien que les quatre textes aient déjà été publiés en français, nous espérons que cette introduction et l'évaluation qu'on y trouve de la situation en Allemagne contribueront à une meilleure information pour les sciences sociales francophones.

\section{Perspectives historiques : l'évolution des nouvelles sciences sociales françaises}

3 La sociologie critique de Pierre Bourdieu, née dans les années soixante, a contribué à mettre en évidence des mécanismes structurels de domination, afin d'en expliquer la reproduction. La puissance du modèle repose sur certaines hypothèses restrictives concernant l'action et sa dynamique, la capacité de l'acteur et notamment son jugement. Dès la fin des années 80 , un renouveau est venu en France d'un courant de recherches sociologiques cherchant à lever ces restrictions et à développer une approche centrée sur une conception plus ouverte de l'action, de son inscription dans une situation, de son incertitude et des capacités cognitives et évaluatives des acteurs. C'est pourquoi ce courant a été désigné comme "sociologie pragmatique». Sans se référer initialement au philosophes pragmatistes américains (Dewey, James, Peirce) mais en bénéficiant de leur héritage via George Herbert Mead, Erving Goffman, ce courant fut d'abord marqué par l'investissement de traditions sociologiques mettant l'accent sur la situation d'action (ethnomethodologie), la phénoménologie de l'action et le jugement dans l'action, des ponts étant établis avec la philosophie de l'action, depuis la philosophie analytique jusqu'à celle de Paul Ricœur, comme en témoigne la série « Raisons Pratiques» (Ed. de l'EHESS) dont le premier numéro de 1990 était intitulé Les formes de l'action ${ }^{2}$.

4 L'ouvrage de Luc Boltanski et Laurent Thévenot d'abord nommé Les économies de la grandeur ${ }^{3}$ puis De la justification ${ }^{4}$, dans son édition définitive, a proposé les fondements théoriques d'une nouvelle sociologie d'abord centrée sur les opérations de critique et de justification soumises au jugement public et impliquant une épreuve de réalité avec un monde matériel environnant. Elle s'est développée d'abord au sein du Groupe de Sociologie Politique et Morale (Ecole des Hautes Etudes en Sciences Sociales et Centre National de la Recherche Scientifique) qu'ils ont créé en 1984 puis dirigé successivement $^{5}$. Un ouvrage récent intitulé Compétences critiques et sens de la justice ${ }^{6}$ présente les itinéraires des deux auteurs après l'ouvrage, ainsi que des éclairages, commentaires et débats autour du programme collectif de recherche qui en a résulté. Rétrospectivement, on peut considérer que cette œuvre a intégré, dans une construction originale, les apports de trois traditions considérées comme profondément divergentes.

5 La notion majeur et dynamique d'épreuve de réalité, moteur de la critique et, à défaut, de sentiment d'injustice, peut être rapportée à l'héritage du pragmatisme américain qui prend au sérieux la dynamique ouverte de l'expérience, ainsi que l'enquête qu'elle suscite (John Dewey). L'incertitude est placée au cœur de l'épreuve. La notion d'épreuve était déjà au cœur de l'autre courant qui a contribué a cette nouvelle sociologie française, celui de l'actor-network-theory, le modèle de Bruno Latour et Michel Callon ayant en outre influencé celui de Boltanski et Thévenot par la place réservée à la dépendance entre humains et non-humains. 
6 La deuxième notion majeure, celle de qualification des personnes et des choses est plutôt d'origine continentale. Empruntée au droit (la qualification des faits est nécessaire pour qu'ils soient pris en compte dans l'application de la loi), elle prend au sérieux les institutions, formalités et classifications qui encadrent les jugements. La perspective de « cognition sociale » avant la lettre qu'avaient ouverte Emile Durkheim et Marcel Mauss dans leur article sur les formes primitives de classifications ${ }^{7}$ a marqué une tradition française puis anglaise (Mary Douglas) de travaux sur les classifications.

7 La troisième notion majeure, celle de bien commun, renoue avec une tradition de philosophie morale et politique avec laquelle les sciences sociales étaient supposées avoir rompu pour établir leur savoir positif. Prenant au sérieux les biens et les maux convoqués dans le jugement des acteurs, elle permet de reconnaître la place de l'évaluation (et non seulement de la cognition) dans la coordination des actions, et d'aborder leur sens critique et leur sentiment d'injustice.

8 Le travail des Economies de la grandeur a constitué une source majeure du courant transdisciplinaire d' "Economie des conventions" qui a réuni des préoccupations d'économistes institutionnalistes et de sociologues. Plusieurs membres fondateurs (François Eymard-Duvernay, André Orléan, Robert Salais, Laurent Thévenot) réunis dans le numéro manifeste de la Revue économique ${ }^{8}$ se trouvaient d'ailleurs initialement en relation à l'Institut national de statistiques et d'études économiques (INSEE) où Alain Desrosières, puis Thévenot, avait mené les travaux pionniers sur les catégorisations ${ }^{9}$. La notion d' »investissement de forme » envisagée comme établissement coûteux d'une forme d'équivalence (code, classement, règle, standard, qualification, certification, coutume, etc..) assurant des économies de coordination ${ }^{10}$ a offert une premier modèle de «mise en forme générale » et d'articulation de «formes conventionnelles » dans les organisations économiques et politiques ${ }^{11}$. Dans sa recherche centrée sur le système financier et ses interférences instables entre coordination par le marché et par la contamination d'opinions, Orléan s'est inspiré de l'approche des conventions d'opinion par John Maynard Keynes ${ }^{12}$. Olivier Favereau a également tiré profit de la réflexion de Keynes sur la pluralité des probables en révélant les apories de l'approche de David Lewis $^{13}$. Professeur à l'Université de Paris X Nanterre, il a dirigé une formation de Master sur l'Economie des conventions et l'économie des institutions. EymardDuvernay, qui a dirigé le Centre d'Etudes de l'Emploi dont Laurent Thévenot était directeur scientifique, a contribué aux développement de recherches de ce courant consacrées aux "modèles d'entreprises » et aux "conventions de qualité " avant de rejoindre Favereau à Paris $\mathrm{X}$ et d'animer un groupe de chercheurs travaillant sur les entreprises, organisations sociales et politiques économiques et sociales, notamment sur les méthodes de recrutement et les intermédiaires du marché du travail. A la suite d'un premier colloque réunissant un ensemble considérable de travaux empiriques du courant EC, il a coordonné la publication de deux volumes présentant ces travaux ${ }^{14}$, deux textes collectifs des fondateurs du courant ainsi que des discussions d'économistes et de sociologues. ${ }^{15}$

\section{Les principaux concepts de la sociologie des conventions}

9 Le concept de convention a une importance stratégique pour bien comprendre les nouvelles sciences sociales françaises ${ }^{16}$. Il faut tout d'abord éviter deux malentendus 
éventuels. (1) Dans la théorie des conventions / EC, le concept de «convention » ne désigne pas uniquement une habitude, un usage, une coutume. La définition que donne Max Weber de la convention en tant que coutume, offre un exemple de cette appréhension limitée de la convention. Elle y figure, au sens de Durkheim, comme un fait social dont la non observation par les membres d'un collectif entraîne des sanctions négatives par ce même collectif ${ }^{17}$. Le concept de convention dans l'EC est plus complexe. (2) Afin de comprendre la contribution de l'EC aux nouvelles sciences sociales françaises, il faut savoir qu'il ne s'agit pas d'une approche unidimensionnelle mais d'un paradigme complet pour l'analyse sociologique et socioéconomique des organisations et institutions sociales, de la cognition sociale, de l'action, de l'interaction et de la coordination ainsi que de la construction sociale des entités et de leurs qualités. En d'autres termes, l'approche de l'EC intègre un réseau dense de concepts reliés les uns aux autres pour former une théorie sociale plus générale.

Les conventions peuvent être appréhendées comme des cadres interprétatifs mis au point et utilisés par des acteurs afin de procéder à l'évaluation des situations d'action et à leur coordination. Les conventions se distinguent des cadres au sens de Goffman, car elles ne sont pas nécessairement liées à une situation. En outre, elles se rapportent à des objets et à des formes cognitives correspondant à des formats d'information ${ }^{18}$. Les acteurs ont recours aux conventions afin de faire valoir et de justifier des qualités et des façons de faire. Le rapport interactif et interprétatif aux objets et le formatage de l'information constituent une façon conventionnelle de qualifier une personne, une action ou un objet. Les conventions sont ainsi des formes culturelles établies collectivement permettant de coordonner et d'évaluer.

11 Eymard-Duvernay et alii (dans ce numéro) montrent que, dans cette approche, les acteurs sont supposés capables d'utiliser les conventions de manière réflexive. Ils sont donc à même de juger de l'adéquation des conventions aux situations réelles et de leur mise en œuvre en situation. En général, les économistes considèrent les conventions comme des solutions au problème de la coordination lorsqu'il y a incertitude (et double contingence). Mais le recours à la convention et son intégration dans l'interaction en situation n'impliquent pas que la coordination réussisse. Les conventions deviennent des ordres de justification ou "grandeurs $»^{19}$ lorsque les acteurs sont critiqués ou critiquent eux-mêmes. Ils s'engagent alors dans une dispute où ils peuvent mobiliser une pluralité de conventions (ordres de justification) discordantes. Le concept de convention n'est donc pas lié à la théorie de l'agir communicationnel de Jürgen Habermas qui développe l'argument selon lequel le «telos de l'accord» réside dans l'utilisation de la langue. Dans la théorie habermassienne, il n'y a finalement qu'une seule convention légitime (celle de la compréhension libérée de toute domination, voir aussi la contribution de Nicolas Dodier dans ce numéro). Mais le concept de convention ne correspond pas non plus à celui d'habitus dans la théorie de Pierre Bourdieu. Les conventions ne sont pas des structures incorporées qui se mettent en œuvre automatiquement. Les acteurs peuvent réfléchir sur l'utilisation des conventions et les conventions sont, d'un point de vue pragmatique, le résultat d'expériences collectives sur les possibilités de coordination face à des problèmes collectifs. Les acteurs créent des conventions dans des situations, et ces conventions peuvent ensuite être stabilisées et consolidées dans des réseaux acteur-objet-concept. A l'inverse, ces conventions peuvent stabiliser ces réseaux. De plus les acteurs sont en mesure d'établir de nouveaux compromis entre les différentes conventions ou de contribuer au changement des 
conventions. Ainsi une dynamique des conventions est introduite à partir d'une perspective pragmatique.

La relation du concept de convention à celui d'institution fait ressortir le caractère duel des conventions. (1) Les conventions peuvent être considérées comme des institutions dans la mesure où elles coordonnent l'action. (2) Mais si l'on confond les institutions avec des règles (comme le fait North ${ }^{20}$ ) alors les conventions ne sont pas des institutions car du point de vue de $\mathrm{EC}$ les règles (et aussi les contrats) sont considérés comme incomplets ${ }^{21}$. Les acteurs doivent interpréter les règles et recourir aux conventions pour faire avec les institutions.

Dans l'analyse établie des institutions économiques, les organisations, les marchés et les réseaux sont couramment considérés comme trois types d'agencements institutionnels ${ }^{22}$. Du point de vue de l'EC chacun de ces agencements n'est pas composé d'une seule forme ou logique de coordination. Les marchés réels, les organisations réelles et les réseaux réels sont des sphères d'interaction en situation (et non pas seulement des structures) dont la régulation réclame de composer avec une pluralité de conventions $^{23}$. Par exemple, l'analyse des marchés peut faire apparaitre des conventions dominantes dans certains segments de marché, ainsi que le rôle de ces conventions dans les relations entre des segments de marché et des organisations.

L'institutionnalisme de Oliver Williamson (et de son prédécesseur Ronald Coase) développé à partir de son approche des coûts de transaction, a contribué à distinguer divers agencements de coordination. Selon cette approche, toute la sphère de l'économie peut être analysée en appliquant la forme de calcul supposée dans la théorie néo-classique, et en y ajoutant les coûts de transaction pour décider quel agencement institutionnel est le plus efficace. A l'inverse, l'EC affirme que les différentes conventions soutiennent différents formes d'évaluation, et donc d'appréciation des coûts et des performances ${ }^{24}$. Chaque convention formate différemment l'information pertinente et construit une forme de qualité (des produits) et de qualification (des personnes $)^{25}$. Du point de vue de la théorie des coûts de transaction, les trois agencements de l'organisation, $\mathrm{du}$ marché et du réseau ne diffèrent que par leurs performances au regard d'une même dimension d'efficacité. Du point de vue de l'EC, la façon de définir et de calculer la valeur varie avec le format d'information et la convention sous-jacente ${ }^{26}$. Eymard-Duvernay montre (dans ce numéro) comment les diverses conventions peuvent coexister dans les entreprises. Les rapports internes et externes peuvent être régulés principalement par l'une ou l'autre des conventions, ou même par une pluralité d'entre elles. Les conventions dans les entreprises productrices sont des conventions de qualité, elles reflètent la façon dont un collectif produit et dont une qualité spécifique est attribuée à un produit pour en faire sa caractéristique reconnue. Cela peut s'effectuer sous une forme techniquement planifiée comme dans le cas de la convention industrielle ou sous la forme de l'habileté du travail artisanal comme dans le cas de la convention dite domestique ${ }^{27}$. Dans les divers marchés de produits, les conventions de qualité peuvent différencier des segments de marché de telle manière qu'une convention de qualité y détermine le régime de production et d'évaluation de la qualitéér.

En caractérisant l'EC de paradigme complet, nous soulignons le fait qu'un groupe croissant de chercheuses et chercheurs français n'a pas seulement réalisé des recherches empiriques dans le domaine de l'économie au cours des deux dernières décennies, mais que cette approche a proposé ses propres fondements 
épistémologiques et théoriques de l'action. Le réseau des concepts esquissés précédemment s'inscrit dans une théorie plus générale de l'interaction (avec des personnes et des objets), de la cognition sociale et de la construction sociale collective (des qualités et des institutions). Pour ce qui est de la théorie de l'action, la théorie des conventions distingue différents niveaux de "régimes». Les conventions sont des cadres collectifs sur lesquels les acteurs prennent appui dans leurs conflits et leurs évaluations en public. Mais il existe des façons de s'engager dans le monde environnant (humain ou non) qui sont moins collectives et plus personnelles, qui assurent des régimes de "convenance" ne permettant pas une coordination d'une aussi large portée, mais gouvernant l'accomplissement d'un projet individuel ou l'appropriation familière dans la relation dynamique entre l'être humain et son environnement ${ }^{29}$. Lorsqu'ils sont mutuels, ces engagements ne permettent qu'une extensions limitée de la coordination. Ici, la théorie proposée offre des développements alternatifs au regard de la catégorie de l'action instrumentale selon Weber et du concept d'habitus chez Bourdieu.

\section{Domaines de recherche et thèmes de recherche : $\mathrm{EC}$ et l'engagement dans l'action.}

Le cadre d'analyse des EC et ses développements récents traversent les frontières entre économique, social et politique, en termes de disciplines mais aussi d'objets et de méthodes de recherche, comme en témoignent les grands chantiers empiriques. Aux outils de la statistique et de l'économétrie qui supposent de saisir les relations à partir de codifications formelles, viennent s'ajouter les méthodes d'enquête et d'analyse supposant une description épaisse (thick) des actions et situations.

\section{Marchés et modèles de production dans l'industrie et les services}

17 La différenciation d'une pluralité de conventions de qualité assise sur des ordres de grandeur a offert un cadre d'analyse systématique pour des objets de recherche qui se trouvent aujourd'hui au cœur d'une sociologie économique de la construction des marchés. Ce cadre permet des comparaisons sur la combinaison de plusieurs qualités en tension dans les façons de créer, de produire («modèles de production » de EymardDuvernay ; «mondes de production » de Salais et Storper), de standardiser et certifier (Kessous, Sylvander) et de mettre à dispositions de clients et d'usager. Les enquêtes portent aussi bien sur l'industrie (biens de consommation : Boisard) que sur les services (banque et produits financiers: Wissler; éducation: Derouet, Normand; santé : Moursli; télémarketing: Kessous et Mallard; logiciels libre: Auray). Reliant les conventions de qualité sur les choses et sur les personnes, le programme empirique de EC a largement couvert le travail dans ses diverses qualités, leur reconnaissance ou déni selon les modes de rémunération, d'évaluation et de recrutement en usage (de Larquier, Marchal). L'évolution du chômage a pu être notamment expliquée par l'écart entre ces conventions de qualité. 


\section{Institutions et politiques publiques, relais des associations et actions politiques dans les dispositifs de régulation, de délibération et de critique}

Exploitant les relations entre les opérations de représentation cognitive (catégorisation), instrumentale (forme matérielle d'équivalence) et politique (élection et grandissement pour contribuer au bien commun), l'analyse de EC permet d'étudier les dispositifs de politiques publiques en action, ainsi que leurs transformations récentes qui font abondamment appel aux associations. Ont été ainsi traitées les politiques du travail (Salais), de l'éducation (Chatel, Normand), de l'action sociale (Breviglieri et Pattaroni), de la santé (Batifoulier et Gadreau) ou de lutte contre la discrimination (Ghirardello, Stavo-Debauge).

Le droit occupe évidemment une place centrale dans ces politiques et peut être luimême décomposé selon les ordres de justifications qu'intègre le jugement et que formalise une pluralité de droits fondamentaux (Lyon-Caen, Thévenot). Les agences de régulation qui se sont substituées à l'Etat ont également fait l'objet d'une analyse mettant en évidence les dynamiques de tension et compromis entre impératifs marchands, industriels et civiques (du service public au service minimum : Mirwasser).

Enfin l'examen du jugement des acteurs et de leurs capacités critiques a permis d'aborder les dispositifs de délibération et de critique associés à la démocratie participative et à la transformation du politique à partir de l'expression du public dans des forums, et autres lieux pour faire entendre une voix (Beuret, Richard-Ferroudji).

\section{L'économique et le politique régis par les instruments de mise en équivalence et d'évaluation : le gouvernement par les normes}

21 Les instruments conventionnels de catégorisation et de comptabilité, depuis la statistique d'Etat (Desrosières, Thévenot) jusqu'aux entreprises (Chiapello), ainsi que les formes de normalisation et de certification de propriétés des produits et de capacités des personnes permettent d'éclairer la transformation historique en cours des régulations qui s'oriente vers un "gouvernement par les normes " (Thévenot). Dès son origine, EC a abordé les politiques à partir de coordinations reposant sur des formes et instruments d'évaluation et de gouvernement, selon une approche largement développée aujourd'hui par les travaux de sciences politiques mettant en avant le gouvernement par les instruments.

\section{Comparaisons historiques et internationales par les conventions en action}

L'analyse de EC à partir des diverses conventions de coordination et de dispositifs conventionnels en action a permis des comparaisons historiques et internationales de large ampleur, notamment dans le domaine du chômage et du travail (Salais, Zimmerman). Les ordres de grandeur (worth) les plus légitimes permettent de décomposer, aux fins de comparaison internationale, des configurations sociales et économiques qui ne sont plus réduites, ni à des caractères très globaux des sociétés ou cultures, ni à des ressources uniquement locales et situationnelles. De larges enquêtes 
collectives internationales et comparatives ont ainsi porté sur conventions de coordination et d'appréciation aux Etats-Unis et en France (Lamont et Thévenot, dir.), ainsi qu'en Russie (Thévenot, dir.). En outre, des recherches ont porté sur les pays émergeants (Cheyns) et notamment les nouvelles formes de gouvernements par «round-tables» suscitées par des acteurs économiques privés en relation avec des ONG.

\section{Des institutions aux garanties en dessous du niveau public : l'extension de EC par la sociologie des engagements}

L'examen des situations d'interaction directe entre l'évaluateur et l'évalué (EymardDuvernay et Marchal) et, plus largement, de la pluralité des engagements qui n'accèdent pas tous aux régimes conventionnels du public, notamment dans les relations de service et les politiques sociales, ont fait l'objet d'une extension du programme EC «en dessous des conventions» (Breviglieri et Stavo-Debauge). Ce " deuxième pluralisme » (Eymard-Duvernay et alii, dans ce numéro) a ouvert l'analyse dynamique des garanties institutionnelles associées au premier pluralisme des grandeurs. L'architecture des "régimes d'engagement» (Thévenot dans ce volume) permet de distinguer de la qualification garantie conventionnellement en public, l'assurance du plan individuel gagée par l'aménagement fonctionnel du monde, et l'aise familière reposant sur l'accommodement $\mathrm{du}$ plus proche, cette dernière offrant une vue nouvelle sur les activités de « care» (Pattaroni).

\section{Pouvoirs et vulnérabilité : de la critique à l'oppression}

Loin d'être une approche conformiste, comme peut le laisser entendre un certain usage $\mathrm{du}$ terme conventionnel, EC offre des instruments d'analyse et d'extension de la critique. Le travail fondateur consacré aux économie de la grandeur portait déjà sur l'analyse des opérations critiques et sur leurs différentes corruptions qui suscitent un sentiment d'injustice, opérations envisagées dans leur réalisation pratique et non seulement dans les principes d'une théorie du juste. Les développements ultérieurs de la sociologie pragmatique ont porté sur la vulnérabilité des personnes et sur les empêchements à faire cheminer, jusqu'à la critique publique, l'expression des maux éprouvés au plus près de la personne. Ces développements offrent une extension de la critique de la domination à partir d'une notion plus large d'oppression, ainsi qu'un dévoilement critique des mécanismes du «gouvernement par l'objectif» qui empêchent la critique (Thévenot 2009a et 2009b et dans ce volume).

\section{La traduction et la réception de la théorie de la convention}

Les quatre textes de ce numéro de TRIVIUM présentent des positions certes contemporaines, mais déjà classiques dans le nouveau domaine de la sociologie pragmatique et de la théorie des conventions. Leur traduction en allemand doit permettre de promouvoir leur réception en Allemagne. 
26 Le problème principal de la réception des nouvelles sciences sociales françaises en Allemagne est la «barrière de la langue ». Aujourd'hui, peu de spécialistes allemand(e)s des sciences sociales lisent des textes français et à l'inverse, peu de spécialistes français(es) des sciences sociales lisent des textes rédigés en allemand. C'est la raison pour laquelle la réception doit emprunter un détour: l'appréhension des sciences sociales outre-rhin passe par le biais des traductions qui existent en anglais; elle est par conséquent très sélective et retardée.

27 Mais il y a un autre problème de réception, ou pour être plus précis une déformation (un biais) de cette réception : les œuvres de Bourdieu rencontrent depuis des années un très large écho, tout particulièrement dans la sociologie allemande. Il est aujourd'hui le sociologue moderne étranger le plus connu en Allemagne. Ceci ne fait pas problème en soi, mais les évolutions survenues après Bourdieu en France et en parallèle à l'œuvre de Bourdieu (et décrites ci-dessus) sont pratiquement ignorées en Allemagne, car l'attention se porte presque exclusivement sur Bourdieu et son école. Il n'y a que quelques exceptions (voir les volumes de Moebius / Peter et de Diaz-Bone ${ }^{30}$ ).

Ces dernières années, quelques travaux de Luc Boltanski et en collaboration ont été traduits en allemand ${ }^{31}$. Mais ici aussi il semble que la réception soit déformée, Boltanski étant essentiellement considéré comme l'ancien collaborateur de Bourdieu. Aujourd'hui les ouvrages de Boltanski sont reçus en Allemagne comme une continuation de l'approche critique du dernier Bourdieu. L'ironie de l'histoire tient à ce que son livre écrit avec Laurent Thévenot «De la justification » a marqué une rupture fondamentale avec Bourdieu et l'école de Bourdieu ${ }^{32}$. La position analytique de Bourdieu consistait à dévoiler, à partir de la position exceptionnelle du sociologue, les mécanismes inconscients opérant derrière les acteurs (comme les mécanismes de l'habitus et de la structure du champ). Contrairement à l'école de Bourdieu, « De la justification » souligne la capacité réflexive des acteurs ordinaires ainsi que ce qui lui fait obstacle. La notion de critique s'en trouve étendue, et démêlée de la position analytique du sociologue procureur des «Lumières modernes". Le tournant pragmatique réalisé avec la théorie des conventions a radicalisé la notion de critique, afin qu'elle puisse être envisagée comme une ressource ordinaire ou comme une compétence des acteurs dans les situations du quotidien. En revanche, le dernier ouvrage de Boltanski $\mathrm{i}^{33}$ semble se réorienter vers une sociologie critique dans le sens de la sociologie d'intervention politique du dernier Bourdieu, sans considérer la contribution de la sociologie pragmatique à l'élargissement de la critique vers des types de vulnérabilité et d'exploitation qui ne sont pas pris en compte par la critique de la domination.

Il faut savoir que depuis le début des années 1990 Boltanski et Thévenot étaient considérés comme persona non grata par l'école de Bourdieu qui ne les citait plus, bien que Bourdieu ait été auparavant le professeur de Boltanski et aussi son ami. La catégorisation du dernier Boltanski peut être utile pour sa réception allemande ${ }^{34}$ mais elle ne l'est certainement pas pour le large domaine de la sociologie pragmatique contemporaine et de la théorie des conventions. Même si « De la justification » est une œuvre fondatrice pour ces nouvelles sciences sociales françaises, le mouvement propre de Boltanski ne s'inscrit pas dans la théorie des conventions. En fait, l'attention croissante dont Boltanski fait l'objet en Allemagne en tant que sociologue critique continuateur de Bourdieu déforme la conception des nouvelles sciences sociales françaises et sa position à leur égard. Leur extension et la place de la théorie des 
conventions comme nouvelle approche pour l'analyse socio-économique des interactions économiques et des institutions économiques est pratiquement ignorée en Allemagne.

Certaines exceptions méritent d'être citées. La revue électronique européenne Economic sociology ${ }^{35}$ a fait paraitre des entretiens avec certains fondateurs de $\mathrm{EC}^{36}$ et un article introductif ${ }^{37}$ (Jagd 2004). L'ouvrage International Encyclopedia of Economic Sociology ${ }^{38}$ a présenté un article introductif sur $\mathrm{EC}^{39}$. Dans le champ allemand de la sociologie économique Jens Beckert, Rainer Diaz-Bone, Jürgen Kädtler, Ursula Mense-Petermann et Sophie Mützel ont commencé à intégrer les concepts de l'EC et à travailler sur cette approche. ${ }^{40}$ La réception de l'EC est en train de s'intensifier en Allemagne ${ }^{41}$.

Dans la sphère anglo-américaine, la situation se présente différemment. Il existe depuis longtemps un article majeur de sociologie expérimentale ouvrant des perspectives sur les pratiques sociales de classification ${ }^{42}$, le travail de Thévenot sur les « investissements dans les formes ${ }^{43}$ ainsi qu'une première publication sur l'utilisation des conventions dans l'analyse des secteurs de l'industrie ${ }^{44}$. L'ouvrage "Monde de productions » de Salais et Storper ${ }^{45}$ a été le premier traduit en anglais (dans une version un peu différente des chapitres $)^{46}$. Quelques années plus tard, Favereau et Lazega ${ }^{47}$ ont publié en anglais un volume sur EC (suite à une discussion entre les représentants de EC et Harrison White). Dans le champ de la sociologie comparative de la culture, une ouvrage collectif, dont quatre chapitres ont été rédigés par Laurent Thévenot et ses collaborateurs, a été publiée ${ }^{48}$ utilisant l'approche tirée de "De la justification ». Depuis, de nombreux articles des représentants des nouvelles sciences sociales françaises sont parus en anglais, présentant la sociologie pragmatique et la théorie des conventions ${ }^{49}$. $\mathrm{Au}$ Danemark Soren Jagd a commencé à travailler en 2007 sur EC..$^{50}$

En 2006, Vando Borghi et Tommaso Vitale ont publié un numéro spécial sur EC dans la revue italienne Sociologia del lavoro $\left(\mathrm{N}^{\circ} 104\right)$ avec des contributions de Bessy, Breviglieri, Eymard-Duvernay, Favereau, Orléan, Salais, Thévenot et des commentaires de Alessandro Pizzorno, Richard Swedberg, David Stark et Charles Tilly. Un recueil italien sur les nouvelles perspectives en sociologie de la culture publié par Marco Santori et Roberta Sassatelli comprend un article de Thévenot et Boltanski comme seule contribution non étasunienne ${ }^{51}$. Un travail plus récent de Thévenot sur « Governing by the objective » est à paraître dans le numéro du $50^{\circ}$ anniversaire de la revue Rassegna Italiana di Sociologia ${ }^{52}$.

En Russie, l'EC a été introduite dans des revues majeures de sciences économiques ${ }^{53}$, de sociologie $e^{54}$, de sciences politiques ${ }^{55}$ et de sciences de la culture ${ }^{56}$. Anton Oleinik a utilisé l'approche de l'EC dans le volume qu'il a publié «Institutional Economics of Russia's Transformations ", paru en russe et en anglais. Il a également rédigé un ouvrage sur le rapport entre la société post-soviétique et les organisations économiques d'une part et la culture carcérale d'autre part (paru en russe et en français en 2001).

\section{Les quatre textes du présent numéro:}

Le texte de Nicolas Dodier traite de l'importance du concept de la convention pour la théorie sociologique de l'action. Cet article montre que la théorie des conventions peut être envisagée comme une contribution à une théorie générale de l'action sociale - et non pas seulement comme le fondement d'une nouvelle approche de sociologie 
économique (ce que l'appellation «économies des conventions » pourrait suggérer de manière trompeuse).

Dans sa contribution à l'analyse des organisations, François Eymard-Duvernay présente la perspective de l'EC sur l'entreprise. Il s'agit d'une théorie de la pluralité des conventions dans les entreprises, qui permettent aux acteurs de réduire la complexité et l'incertitude de la coordination collective dans la production et la distribution.

La contribution de Laurent Thévenot présente le coeur d'un nouveau développement analytique après «De la justification ». La pluralité des "Régimes d'engagement " éclaire les façons de s'impliquer qui confèrent capacité et pouvoir en deçà du niveau le plus public des qualifications basées sur les conventions. Cette contribution donne aussi un aperçu de recherches empiriques éclairant des transformations micro et macro des politiques sociales et éducatives, ainsi que des pratiques favorisées par les nouvelles technologies de l'information et de la communication.

quatrième texte est l'introduction d'une série deux volumes présentant la recherche récente dans le vaste domaine de l'EC, au milieu de cette décennie. Les fondateurs de cette approche présentent les principaux concepts de l'EC et esquissent l'intégration théorique et l'architecture de l'EC.

De notre point de vue la série d'articles que nous présentons ici permet de donner aux lectrices et lecteurs un aperçu de l'étendue conceptuelle de la théorie des conventions mais aussi de son évolution. Nous espérons ainsi faire progresser la réception internationale de cette approche innovatrice et transdisciplinaire.

\section{BIBLIOGRAPHIE}

Beckert, J. / Zafirovsky, M. (ed.) (2006) : International encyclopedia of economic sociology. London, Routledge.

Boltanski, L. (1982) : Les cadres, Paris, Éditions de Minuit.

Boltanski, L. (1990) : Die Führungskräfte, Francfort, Campus.

Boltanski, L. (2004) : La condition foetale, Paris, Gallimard.

Boltanski, L. (2007) : Über die Abtreibung, Francfort, Suhrkamp.

Boltanski, L. (2009) : De la critique. Précis de sociologie de l'emancipation, Paris, Gallimard.

Boltanski, L. / Chiapello, E. (1999) : Le nouvel esprit du capitalisme, Paris, Gallimard.

Boltanski, L. / Chiapello, E. (2003) : Der neue Geist des Kapitalismus, Constance, UVK.

Boltanski, L. / Thévenot, L. (1983) : « Finding one's way in social space : A study based on games »,Social Science Information, 22 (4/5), p. 631-680.

Boltanski, L. / Thévenot, L. (1987) : Les économies de la grandeur, Paris, Presses Universitaires de France et Centre d'Etude de l'Emploi. 
Boltanski, L. / Thévenot, L. (ed.) (1989) : Justesse et justice dans le travail, Paris, PUF (Cahiers du Centre d'Etudes de l'Emploi, 33).

Boltanski, L. / Thévenot, L. (1991) : De la justification. Les économies de la grandeur, Paris, Gallimard. Boltanski, L. / Thévenot, L. (2007) : Über die Rechtfertigung, Hambourg, Hamburger Edition. Borghi V. / Vitale T. (ed.) (2007) : Le convenzioni del lavoro, il lavoro delle convenzioni, numero monografico di Sociologia del Lavoro, 102, Milan, Franco Angeli.

Breviglieri, M. / Lafaye, C. / Trom, D. (ed.) (2009) : Compétences critiques et sens de la justice, Paris, Economica.

Corcuff, P. (2007) : Les nouvelles sociologies - Entre le collectif et l'individuel, Paris, Armand Colin.

Desrosières, A. (1987 [1977]) : « Eléments pour l'histoire des nomenclatures

socioprofessionnelles ", dans : Affichard, J. (ed.) : Pour une histoire de la statistique, tome 1, Paris, INSEE, p. 155-231.

Desrosières, A. / Thévenot, L. (1988) : Les catégories socioprofessionnelles, Paris, La Découverte.

Dequech, D. (2006) : « Cognition and valuation : some similarities and contrasts between institutional economics and the economics of convention ", Journal of Economic Issues, 39 (2), p. $465-473$.

Diaz-Bone, R. (2006) : "Wirtschaftssoziologische Perspektiven nach Bourdieu in Frankreich », dans : Florian, M. / Hillebrandt, F. (ed.) : Pierre Bourdieu : Neue Perspektiven für die Soziologie der Wirtschaft. Wiesbaden, VS-Verlag, p. 43-71.

Diaz-Bone, R. (2009) : «Économie des conventions », dans : Beckert, J. / Deutschmann, Ch. (ed.) (2009) : Wirtschaftssoziologie. Kölner Zeitschrift für Soziologie und Sozialpsychologie, Sonderheft 49, Wiesbaden, VS-Verlag, p. 176-193.

Diaz-Bone, R. (à paraître) : Économie des conventions, Wiesbaden, VS-Verlag.

Diaz-Bone, R. (ed.) (à paraître) : Soziologie der Konventionen, Francfort, Campus.

Dosse, F. (1995) : L'empire du sens. L'humanisation des sciences humaines, Paris, La Découverte.

Dosse, F. (1998) : Empire of meaning : the humanization of the social sciences, Minneapolis, Univ. of Minnesota Press.

Dupuy, J.-P. et al. (1989) : Revue économique, numéro spécial L'économie des conventions, 40 (2).

Durkheim, E. / Mauss, M. (1971 [1903]) : « De quelques formes primitives de classification. Contribution à l'étude des représentations collectives » (Année sociologique, 6, 1903) republié dans : Mauss, M. : Essais de sociologie, Paris, Ed. de Minuit (Points).

Eymard-Duvernay, F. (1989) : «Conventions de qualité et forms de coordination », Revue économique, 40 (2), p. 329-359.

Eymard-Duvernay, F. (ed.) (2006) : L'économie des conventions,Paris, La Découverte, tome I : Méthodes et résultats, tome II : Développements, Paris, La Découverte.

Favereau, O. / Lazega, E. (ed.) (2002) : Conventions and structures in economic organization, Cheltenham, Edward Elgar.

Granovetter, M. (1985) : « Economic action : the Problem of embeddedness », American Journal of Sociology, 91 (3), p. 481-510. 
Jagd, P. (2004) : « Laurent Thévenot and the French convention school. A short introduction », Economic Sociology - European Electronic Newsletter, 5 (3), p. 2-9

(http://econsoc.mpifg.de/newsletter/newsletter_archive.asp).

Jagd, P. (2007) : « Economics of convention and new economic sociology », Current Sociology, 55 (1), p. 75-91.

Lamont, M. / Thévenot, L. (ed) (2000) : Rethinking comparative sociology. Repertoires of evaluation in France and the United States, Cambridge, Cambridge University Press.

Moebius, P. / Peter, L. (ed.) (2004) : Französische Soziologie der Gegenwart, Constance, UVK.

North, D. (1990) : Institutions, institutional change and economic performance, Cambridge, Cambridge University Press.

Oleinik, A. (2001) : Criminalité organisée, prison et sociétés post-soviétiques, Paris, Torino, L'Harmattan (préface d'Alain Touraine).

Oleinik, A. (ed.) (2005) : The Institutional Economics of Russia's Transformations, Aldershot (UK), Ashgate.

Orléan, A. (ed.) (2004 [1994]) : Analyse économique des conventions, $2^{\mathrm{e}}$ édition avec post-face additionnelle de André Orléan, Paris, PUF (collection Quadrige).

Orléan, A. (2005) : « La sociologie économique et la question de l'unité des sciences sociales », L'Année Sociologique, 55 (2), p. 279-305.

Salais, R. (1989) : «L'analyse économique des conventions du travail », Revue économique, 40 (2), p. $199-240$.

Salais, R. (2007) : « Ökonomie der Konventionen - mit einer Anwendung auf die Arbeitswelt », dans : Beckert, J. / Diaz-Bone, R. / Ganßmann, H. (ed.) (2007) : Märkte als soziale Strukturen, Francfort, Campus, p. 95-112.

Salais, R. (2008) : « Robert Salais answers five questions about economic sociology. Robert Salais interviewed by Rainer Diaz-Bone in Berlin », Economic Sociology - European Electronic Newsletter, 9 (2), p. 16-23.

Salais, R. / Storper, M. (1992) : « The Four Worlds of Contemporary Industry », Cambridge Journal of Economics, 16 (2), p. 169-194

(http://econsoc.mpifg.de/newsletter/newsletter_archive.asp).

Salais, R. / Storper, M. (1993) : Les mondes de production, Paris, EHESS.

Steiner, P. (2009) : «Economic sociology in France : Interview with Philippe Steiner », Economic Sociology - European Electronic Newsletter, 10 (3), p. 29-33

(http://econsoc.mpifg.de/newsletter/newsletter_archive.asp).

Storper, M. / Salais, R. (1997) : Worlds of production, Cambridge, Harvard University Press.

Thévenot, L. (1979) : « Une jeunesse difficile. Les fonctions sociales du flou et de la rigueur dans les classements ", Actes de la recherche en sciences sociales, 26-27, p. 3-18.

Thévenot, L. (1984) : « Rules and implements : investment in forms », Social Science Information, 23 (1), p. 1-45.

Thévenot, L. (1989) : «Équilibre dans un univers complexe », Revue économique, 40 (2), p. 147-197.

Thévenot, L. (2001) : « Organized complexity : Conventions of coordination and the composition of economic arrangements », European Journal of Social Theory, 4 (4), p. 405-425. 
Thévenot, L. (2004) : «The French convention school and the coordination of economic action. Laurent Thévenot interviewed by Søren Jagd », Economic Sociology - European Electronic Newsletter, 5 (3), p. 10-16

(http://econsoc.mpifg.de/newsletter/newsletter_archive.asp).

Thévenot L. (2005) : « The Two Bodies of May “68 : In Common, in Person », dans : Sica A. / Turner, S. (ed.) : The Disobedient Generation : Social theorists in the sixties, Chicago, University of Chicago Press, p. 252-271.

Thévenot, L. (2006a) : « Laurent Thévenot answers ten questions about economic sociology », Economic Sociology - European Electronic Newsletter, 8 (1), p. 36-40

(http : / / econsoc.mpifg.de / newsletter / newsletter_archive.asp).

Thévenot, L. (2006b) : « Convention school », dans : Beckert, J. / Zafirovski, M. (ed.) : International Encyclopedia of Economic Sociology, London, Routledge, p. 111-115.

Thévenot, L. (2007) : « The plurality of cognitive formats and engagements : Moving between the familiar and the public », European Journal of Social Theory, 10 (3), p. 409-423.

Thévenot, L. (2009a) : « Governing Life by Standards. A View from Engagements », Social Studies of Science, 39 (5), p. 793-813.

Thévenot, L. (2009b) : « Des autorités à l'épreuve de la critique. L'opprimant du "gouvernement par l'objectif" ", contribution au cinquantième anniversaire de la Rassegna Italiana di Sociologia.

Thévenot, L. / Boltanski, T. (2009) : «Verso una sociologia della capacità critica », dans : Santori, M. / Sassatelli, R. (ed.) : Studiare la cultura. Nuove prospettive sociologiche, Bologna, Il Mulino, p. 309-329.

Weber, M. (1972) : Wirtschaft und Gesellschaft. $5^{\mathrm{e}}$ ed., Tübingen, Mohr.

Williamson, O. (1985) : The economic institutions of capitalism, New York, Free Press.

\section{NOTES}

1. Dosse (1995 [angl. 1998]).

2. Ecrivent dans cette première livraison qui marque ce "tournant pragmatique" les sociologues français Bernard Conein, Nicolas Dodier, Patrick Pharo, Louis Quéré et Laurent Thévenot qui seront de fréquents contributeurs de la série. Sont également traduits des textes d'ethnométhodologues de langue anglaise (Lucy Suchman, Wes Sharrock et Rod Watson) et présentés des textes de philosophes de l'action (Elisabeth Anscombe, Jean-Luc Petit). L'ouvrage de l'historien François Dosse replace ce mouvement dans un tableau plus général des sciences humaines et de la philosophie remaniées par "l'empire du sens", pour reprendre le titre de son ouvrage $(1995,1998)$.

3. Boltanski / Thévenot (1987).

4. Boltanski / Thévenot (1991 tr. 2007).

5. Sur cette sociologie située dans un panorama plus ample, voir Corcuff 2007.

6. Breviglieri et al. (ed.) 2009.

7. Durkheim / Mauss (1971 [1903]).

8. Dupuy et al. (1989).

9. Desrosières (1987 [1977]), Thévenot (1979), Desrosières / Thévenot (1988).

10. Thévenot (1984). 
11. Remarquons que l'“économie" des formes conventionnelles et des grandeurs désigne donc des économies [economies en anglais] de coordination, notamment cognitives, et non pas seulement le domaine de l'économie [economics en anglais].

12. Voir son introduction au volume dont il a dirigé la publication (Orléan 2004 [1994]) ainsi que Orléan 2005.

13. Olivier Favereau prépare un ouvrage d'introduction à EC à paraitre à La Découverte (collection Repères).

14. Eymard-Duvernay (2006).

15. Un récente semaine de Cerisy a été l'occasion de discuter une nouvelle série de recherches sur le système financier et sa crise, les organisations du marché du travail, les politiques économiques et sociales et leurs instruments d'évaluation, et plus généralement la contribution à la relance de la critique que permet l'EC. Une nouvelle publication est en préparation.

16. Dosse (1995 [angl. 1998]), Corcuff (2007).

17. Weber (1972), p. 17.

18. Thévenot (1984).

19. Boltanski/Thévenot (1991 [tr. 2007]).

20. North (1990).

21. Voir Eymard-Duvernay et al. dans ce numéro, ainsi que Eymard-Duvernay dans ce numéro, Salais (2008).

22. Williamson (1985), Granovetter (1985).

23. Salais (1989), (2007), Thevenot (1989), (2001), Eymard Duvernay (1989 et dans ce numéro).

24. Voir aussi Dequech (2005).

25. Thévenot (2007).

26. Thévenot (1989), Salais / Storper (1993).

27. Voir Diaz-Bone (2009).

28. Favereau / Lazega (2002).

29. Voir Thévenot (2009a, 2009b et dans ce numéro).

30. Moebius / Peter (2004), Diaz-Bone (2006).

31. Boltanski (1982 [tr. 1990]), Boltanski (2004 [tr. 2007]), Boltanski / Chiapello (1999 [tr 2003]).

32. La rupture avec la sociologie de Bourdieu ne porte pas sur l'importance de la critique pour les sciences sociales mais sur la nécessité de prendre en considération les conditions ordinaires (non savantes) de la critique. Alors que Boltanski fut longtemps un collaborateur majeur du Centre de Sociologie Européenne (dirigé par Bourdieu), Thévenot qui est de neuf ans son cadet n'y fut lié qu'un temps court, mais très important pour sa formation (Thévenot 1979, 2005).

33. Boltanski (2009).

34. Basaure (2008).

35. La revue est produite au Max-Planck-Institute for the Study of Societies at Cologne (http:// econsoc.mpifg.de/newsletter/newsletter_archive.asp)

36. Thévenot (2004), Salais (2008).

37. Jagd (2004).

38. Beckert / Zafirovski (2006).

39. Thévenot (2006b).

40. Diaz-Bone (2009), Diaz-Bone (à venir).

41. Pour la première traduction d'une contribution française, voir Salais (2007).

42. Boltanski / Thévenot (1983).

43. Thévenot (1984).

44. Salais / Storper (1992).

45. Salais / Storper (1993).

46. Storper / Salais (1997).

47. Favereau / Lazega (2002). 
48. Lamont / Thévenot (2000).

49. Voir Thévenot (2007) et la contribution de Dodier dans cette édition, qui est parue en 1995 en traduction anglaise

50. Jagd (2007).

51. Thévenot / Boltanski (2009).

52. Thévenot (2009b).

53. Vaprosi Ekonomiki, 10, 1997 ; 2010 à paraître.

54. Journal sotsiologi i sotsialnoy antropologi, III (3), 2000 ; Sotsiologitcheski Journal, 1-2, 2006

55. Neprikosnoveny Zapas, 3 (35), 2004.

56. Novoye Literaturnoye Obrazovanie, 77, 2006 et 100, 2010.

\section{AUTEURS}

\section{RAINER DIAZ-BONE (UNIVERSITÉ DE LUCERNE)}

Professeur de sociologie, Université de Lucerne. Pour plus d'informations, cliquez ici.

\section{LAURENT THÉVENOT (EHESS PARIS)}

Directeur de recherche, École des Hautes Études en Sciences Sociales (EHESS, Groupe de Sociologie Politique et Morale, GSPM) et administrateur de l'Institut National de la Statistique et des Études Économiques, Paris (INSEE, Laboratoire de Sociologie Quantitative). Pour plus d'informations, cliquez ici. 